OPEN ACCESS

Edited by:

Anthonie J. Van Der Wekken, University Medical Center Groningen,

Netherlands

Reviewed by:

Timothy F. Burns,

University of Pittsburgh, United States

Youxin Ji,

Qingdao Central Hospital, China

${ }^{*}$ Correspondence:

Wei Dong

dr.dongwei@sdu.edu.cn

Specialty section:

This article was submitted to

Thoracic Oncology,

a section of the journal

Frontiers in Oncology

Received: 11 August 2021 Accepted: 29 September 2021

Published: 20 October 2021

Citation:

Wang Y, Liu S, Feng A, Luo H, Hu J, Wang $K$ and Dong W (2021)

Identification of a Rare EGFR T790/

Mutation in Lung Adenocarcinoma

Sensitive to Osimertinib.

Front. Oncol. 11:727312.

doi: 10.3389/fonc.2021.727312

\section{Identification of a Rare EGFR T790I Mutation in Lung Adenocarcinoma Sensitive to Osimertinib}

\author{
Yu Wang ${ }^{1}$, Songtao Liu ${ }^{1}$, Alei Feng ${ }^{1}$, Huan Luo ${ }^{2}$, Jinwei Hu${ }^{2}$, \\ Kai Wang ${ }^{2}$ and Wei Dong ${ }^{3,4 *}$ \\ 1 Department of Oncology, Shandong Provincial Hospital Affiliated to Shandong First Medical University, Jinan, China, ${ }^{2}$ OrigiMed \\ Inc., Shanghai, China, ${ }^{3}$ Department of Thoracic Surgery, Shandong Provincial Hospital, Cheeloo College of Medicine, Shandong \\ University, Jinan, China, ${ }^{4}$ Department of Thoracic Surgery, Shandong Provincial Hospital Affiliated to Shandong First Medical \\ University, Jinan, China
}

Estimated glomerular filtration rate (EGFR)-sensitive mutations are extremely important for targeted treatment strategies in lung cancer. Osimertinib can effectively inhibit the activity of EGFR-sensitive mutations, including the T790M mutation. However, the efficiency of osimertinib for rare mutation types of T790 is unclear. Here, we report the case of a Chinese patient with lung adenocarcinoma (LADC) harboring a T790l mutation who achieved significant benefits from osimertinib treatment.

Keywords: lung adenocarcinoma (AC), T790I, osimertinib, EGFR, target therapy

\section{INTRODUCTION}

Estimated glomerular filtration rate (EGFR)-tyrosine kinase inhibitors (TKIs) are a standard treatment option for patients harboring activating EGFR mutations, and several TKIs that specifically target EGFR have been approved by the FDA (1). However, many patients develop acquired drug resistance because of the EGFR T790M mutation (2). Although osimertinib can effectively inhibit the activity of the EGFR-sensitive mutation T790M (3), the efficiency of osimertinib for the rare mutation type T790 is unclear; therefore, the recognition and clinical evidence of rare mutations is essential for precision oncology. Hence, we report a Chinese patient with lung adenocarcinoma (LADC) with a novel EGFR T790I mutation, which is a rare predicted first-generation TKI-resistance mutation. He achieved significant benefits accompanied by undetectable T790I expression after osimertinib treatment (Figure 1A).

\section{PATIENT AND SEQUENCING}

This study was approved by the institutional review board and conducted following the tenets of the Declaration of Helsinki. Informed consent was obtained from the patient. Formalin-fixed paraffinembedded (FFPE) tumors and matched blood samples from a 61-year-old Chinese male with LADC were collected and transferred to OrigiMed (Shanghai, China) for genetic alteration detection.

Genomic DNA of tumor samples and white blood cells from matched blood was extracted from the FFPE tumor and blood samples using the QIAamp DNA FFPE Tissue Kit and QIAamp DNA Blood Midi Kit (Qiagen, Hilden, Germany) according to the manufacturer's instructions. The testing was carried out in a laboratory certified by the College of American Pathologists and Clinical Laboratory Improvement Amendments. 

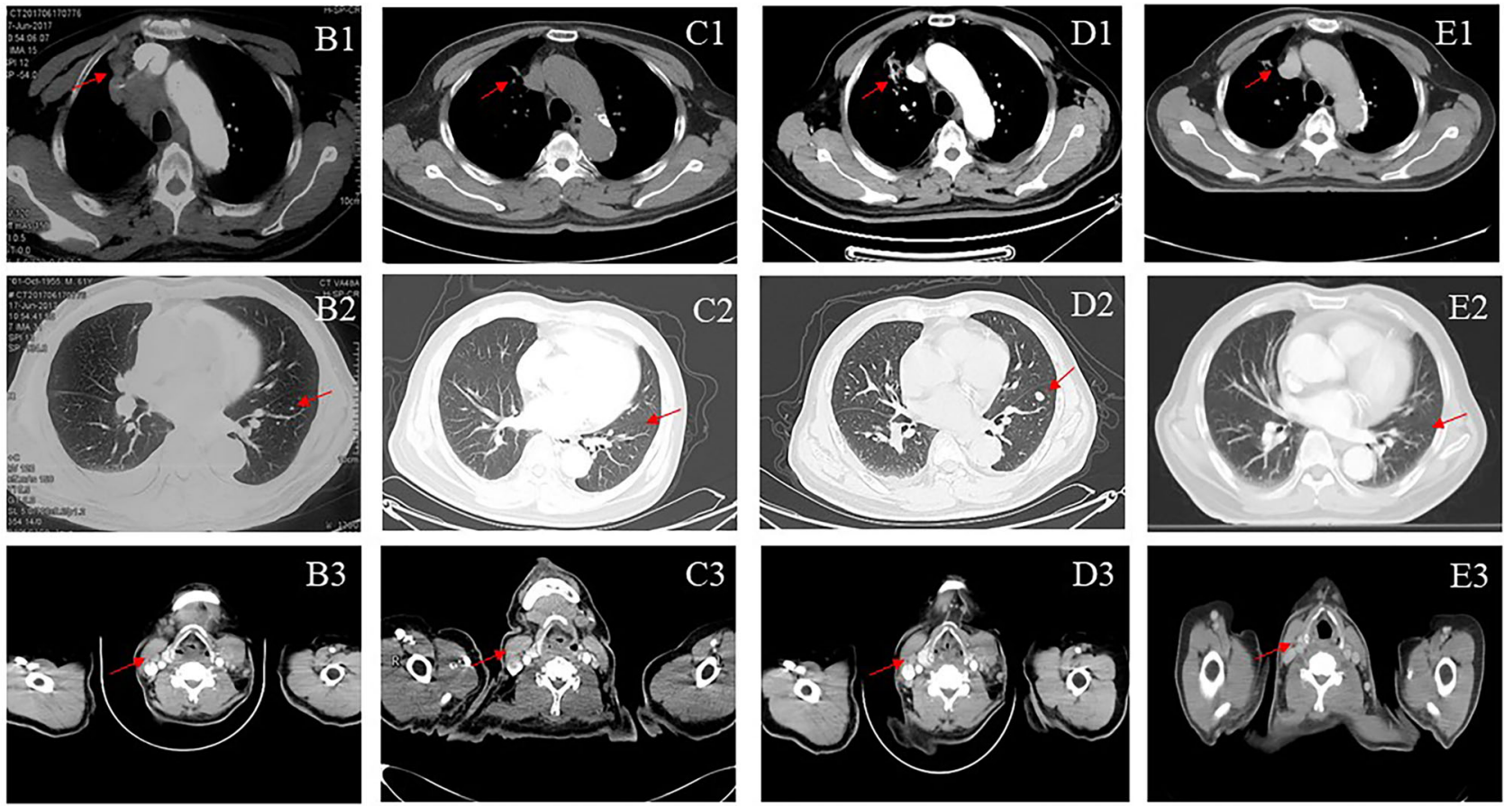

Baseline

Icotinib PR

Icotinib PD

Osimertinib PR

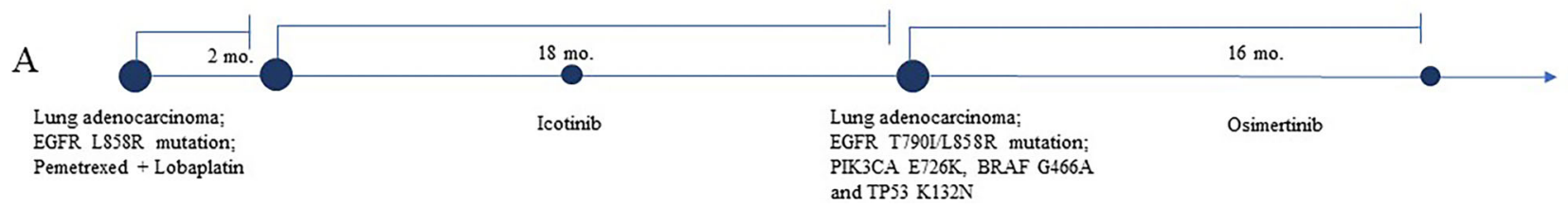

FIGURE 1 Computed tomographic (CT) images (B-E) of the patient during treatment course (A). Baseline images at diagnosis (June 2017) (B1-3). Images after icotinib treatment (C1-3). The patient's upper lobe of the right lung, mediastinal and right hilar lymph nodes, bilateral pulmonary nodules, and right supraclavicular region were smaller. Eighteen months after icotinib treatment disease progression (D1-3). The patient achieved a sustainable clinical benefit from osimertinib treatment (E1-3).

\section{RESULTS}

The patient was a 61-year-old Chinese man without a history of smoking or family history of lung cancer. He had been receiving systemic treatment for coronary heart disease for 13 years. This patient was admitted with a 2-month history of chest tightness and asthmatic and irregular coughing in June 2017. Chest computed tomography (CT) revealed a space-occupying lesion in the upper lobe of the right lung, which invaded the mediastinal and right hilar lymph nodes and right supraclavicular region (Figure 1B). Right supraclavicular lymph node biopsy was performed, and pathological examination allowed diagnosis of lung adenocarcinoma T2N3M1a (Figure 2A), and immunohistochemistry (IHC) revealed the tumor to be NapsinA+, TTF-1+, CK7+, CD10-, and vimentin+. The EGFR L858R mutation was detected using a conventional amplification-refractory mutation system. The patient initially received intravenous pemetrexed combined with lobaplatin but could not tolerate after one cycle; thus, he was administered icotinib (125 mg three times daily) (Figure 1C) in August 2017.
In February 2019, after treatment with icotinib for 18 months, a CT scan showed disease progression (Figure 1D) with multiple bilateral pulmonary nodules and multiple mediastinal and lymph node metastases. A lung biopsy after radiographic disease progression showed adenocarcinoma again (Figure 2B). After obtaining consent from the patient, his FFPE tumor sample was sent to the OrigiMed (Shanghai, China) for next-generation sequencing analysis with a YuanSu ${ }^{\mathrm{TM}}$ panel (4). The T790I (c.2369_2370delinsTA) mutation, a rare variant which causes a threonine (T) to isoleucine (I) missense mutation at codon 790 , was identified (Figure 3). Other mutations were also detected, including EGFR L858R, PIK3CA E726K, BRAF G466A, and TP53 K132N. The patient was started on osimertinib $(80 \mathrm{mg}$ once daily) treatment in March 2019 with good tolerance and treatment for 16 months. After a follow-up in July 2020, a partial response (RECIST 1.1) was obtained for osimertinib treatment, and the mediastinal and lymph nodes disappeared (Figure 1E). The next-generation sequencing results of cell-free DNA showed that both the EGFR T790I and L858R mutations were negative. 


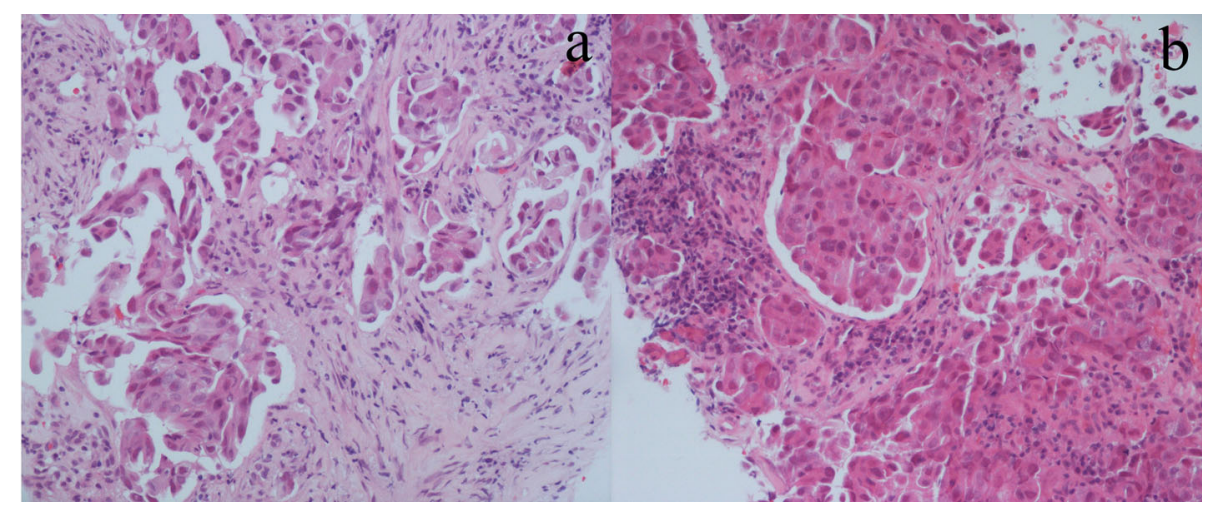

FIGURE 2 | Hematoxylin and eosin staining of the first (A) and repeated (B) biopsy specimens.

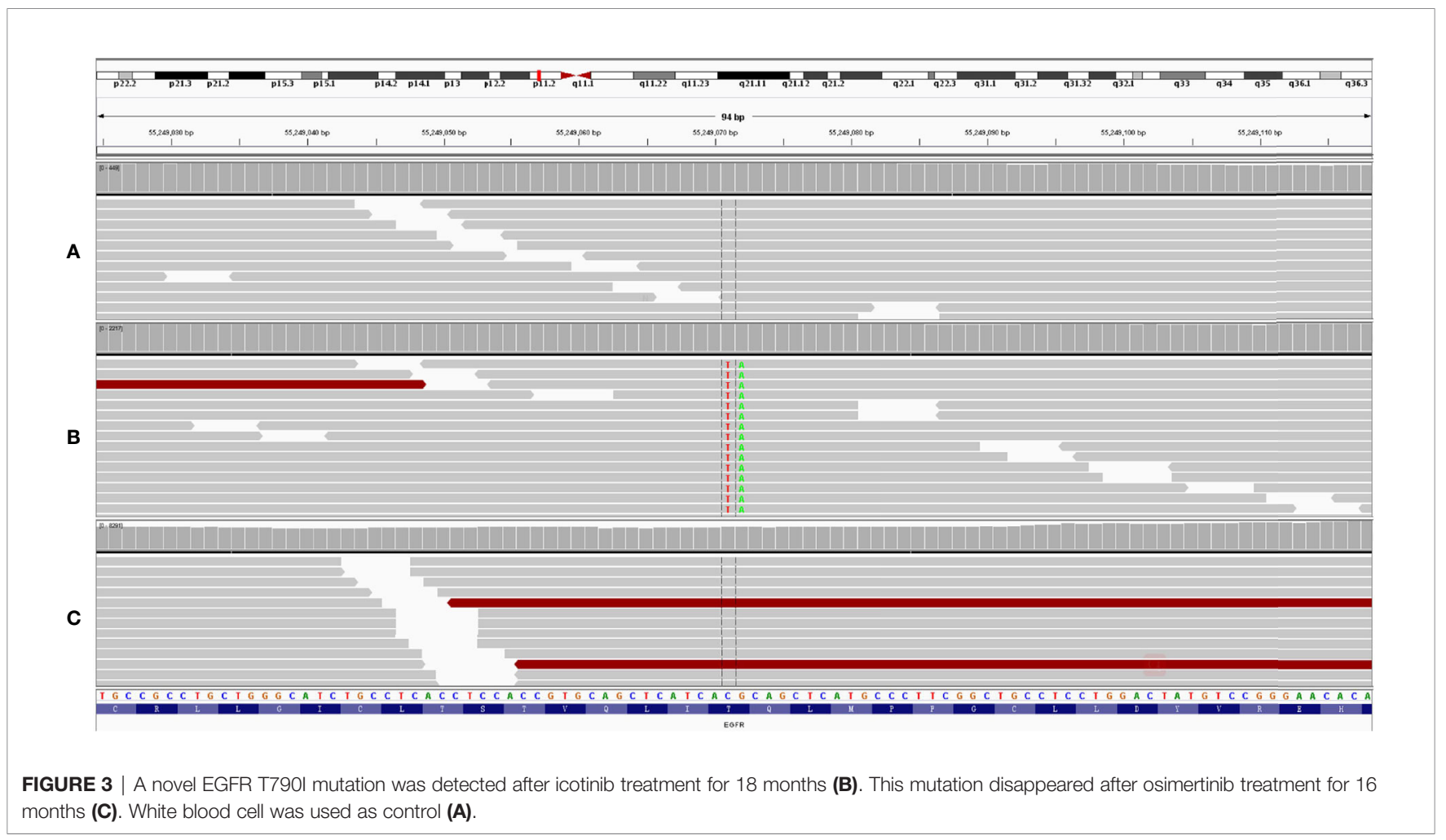

Up to the date of this report, the patient is still receiving osimitinib treatment, and his condition is stable without significant progress.

\section{DISCUSSION}

The "gatekeeper" residue threonine 790 is an important determinant of inhibitor specificity in the ATP-binding pocket as increased ATP affinity is the primary mechanism by which the T790M mutation confers drug resistance $(5,6)$. T790I may also act as a "gatekeeper" mutation to activate kinase activity in receptor tyrosine kinases. A computational exploration of "gatekeeper" mutations in EGFR revealed that EGFR T790M and EGFR T790I stabilize both the active and inactive forms of the kinases (7). The oncogenic activity of the T790I mutation has previously been reported in $\mathrm{Ba} / \mathrm{F} 3$ cells (8) but has not been observed in TKI-refractory lung cancers in clinics. A previous study revealed that osimertinib can inhibit the growth of transgenic lung cancer mouse models harboring mouse EGFR exon19 deletion (mDEL) tumors with T790I mutation; however, these tumors acquired resistance to osimertinib and regrew 2 months after treatment (9). In our case, after disease progression was caused by the development of drug resistance to a first- 
generation EGFR TKI, the patient achieved partial response and progression-free survival after osimertinib treatment. Although osimertinib can effectively inhibit the activity of EGFR-sensitive mutations and T790M (10), a number of other tertiary EGFR mutations have been observed to confer osimertinib resistance. Similar to EGFR T790M, the T790I mutation confers resistance to icotinib; however, in this case, T790I appears to have a weaker resistance mutation than $\mathrm{T} 790 \mathrm{M}$ and loses activation of both EGFR and downstream signaling following osimertinib treatment.

This is the first report of an LADC patient with EGFR T790I mutation who achieved a good response with osimertinib, thereby presenting the first clinical evidence of the efficacy of osimertinib, targeting T790I, which indicated that T790I is a candidate osimertinib-sensitive mutation. The result is an individualized treatment, maybe a limitation of this study, but also demonstrates the precision therapies being crucial for optimal individualized management and thus elevating the survival rate. Furthermore, our findings highlight the importance of dynamic genetic monitoring to tailor precision therapies to improve clinical outcomes.

\section{DATA AVAILABILITY STATEMENT}

The datasets presented in this study can be found in online repositories. The names of the repository/repositories and accession number(s) can be found in the article/supplementary material.

\section{ETHICS STATEMENT}

The studies involving human participants were reviewed and approved by the ethics committee of Shandong Provincial

\section{REFERENCES}

1. Ke EE, Wu Y-L. EGFR as a Pharmacological Target in EGFR-Mutant NonSmall-Cell Lung Cancer: Where Do We Stand Now? Trends Pharmacol Sci (2016) 37:887-903. doi: 10.1016/j.tips.2016.09.003

2. Yu HA, Arcila ME, Rekhtman N, Sima CS, Zakowski MF, Pao W, et al. Analysis of Tumor Specimens at the Time of Acquired Resistance to EGFRTKI Therapy in 155 Patients With EGFR-Mutant Lung Cancers. Clin Cancer Res (2013) 19:2240-7. doi: 10.1158/1078-0432.CCR-12-2246

3. Cross DAE, Ashton SE, Ghiorghiu S, Eberlein C, Nebhan CA, Spitzler PJ, et al. AZD9291, an Irreversible EGFR TKI, Overcomes T790M-Mediated Resistance to EGFR Inhibitors in Lung Cancer. Cancer Discov (2014) 4:1046-61. doi: 10.1158/2159-8290.CD-14-0337

4. Cao J, Chen L, Li H, Chen H, Yao J, Mu S, et al. An Accurate and Comprehensive Clinical Sequencing Assay for Cancer Targeted and Immunotherapies. Oncologist (2019) 24:1294-302. doi: 10.1634/theoncologist.2019-0236

5. Yun C-H, Mengwasser KE, Toms AV, Woo MS, Greulich H, Wong K-K, et al. The T790M Mutation in EGFR Kinase Causes Drug Resistance by Increasing the Affinity for ATP. Proc Natl Acad Sci USA (2008) 105:2070-5. doi: 10.1073/pnas.0709662105

6. Singh M, Jadhav HR. Targeting non-Small Cell Lung Cancer With SmallMolecule EGFR Tyrosine Kinase Inhibitors. Drug Discov Today (2018) 23:745-53. doi: 10.1016/j.drudis.2017.10.004

7. Kannan S, Fox SJ, Verma CS. Exploring Gatekeeper Mutations in EGFR Through Computer Simulations. J Chem Inf Model (2019) 59:2850-8. doi: 10.1021/acs.jcim.9b00361

8. Azam M, Seeliger MA, Gray NS, Kuriyan J, Daley GQ. Activation of Tyrosine Kinases by Mutation of the Gatekeeper Threonine. Nat Struct Mol Biol (2008) 15:1109-18. doi: 10.1038/nsmb.1486
Hospital. The patients/participants provided their written informed consent to participate in this study. Written informed consent was obtained from the individual(s) for the publication of any potentially identifiable images or data included in this article.

\section{AUTHOR CONTRIBUTIONS}

WD and YW had full access to all of the data in the manuscript and take responsibility for the integrity of the data and the accuracy of the data analysis. Concept and design: all authors. Acquisition, analysis, and interpretation of data: all authors. Manuscript drafting: WD and YW. Critical revision of the manuscript for important intellectual content: all authors. Statistical analysis: YW and AF. Supervision: WD. All authors contributed to the article and approved the submitted version.

\section{FUNDING}

This work was supported by the National Natural Science Foundation of China (81802282) and the Jinan Science and Technology Development Program (201907112).

\section{ACKNOWLEDGMENTS}

We would like to thank Editage (www.editage.cn) for English language editing. The authors would like to thank the patient for providing written informed consent for publication and all the research staff involved in this case study.

9. Ohashi K, Higo H, Makimoto G, Kudo K, Nishii K, Ninomiya K, et al. Abstract 1160: The Novel Osimertinib Resistant Lung Cancer Mice Model Harboring EGFR Mutations Driven by the SP-C Promoter. Cancer Res (2018) 78:1160. doi: 10.1158/1538-7445.AM2018-1160

10. Ramalingam SS, Vansteenkiste J, Planchard D, Cho BC, Gray JE, Ohe Y, et al. Overall Survival With Osimertinib in Untreated, -Mutated Advanced NSCLC. New Engl J Med (2020) 382:41-50. doi: 10.1056/NEJMoa1913662

Conflict of Interest: Author HL, JH and KW were employed by the OrigiMed Inc. The remaining authors declare that the research was conducted in the absence of any commercial or financial relationships that could be construed as a potential conflict of interest.

Publisher's Note: All claims expressed in this article are solely those of the authors and do not necessarily represent those of their affiliated organizations, or those of the publisher, the editors and the reviewers. Any product that may be evaluated in this article, or claim that may be made by its manufacturer, is not guaranteed or endorsed by the publisher.

Copyright (c) 2021 Wang, Liu, Feng, Luo, Hu, Wang and Dong. This is an openaccess article distributed under the terms of the Creative Commons Attribution License (CC BY). The use, distribution or reproduction in other forums is permitted, provided the original author(s) and the copyright owner(s) are credited and that the original publication in this journal is cited, in accordance with accepted academic practice. No use, distribution or reproduction is permitted which does not comply with these terms. 